\title{
4. Una mirada a la sociedad de la transparencia en Latinoamérica
}

Narcisa Jessenia Medranda Morales ${ }^{1}$ Victoria Dalila Palacios Mieles ${ }^{2}$

\section{Resumen}

Esta investigación busca analizar desde un punto de vista crítico, la efectividad de la legislación sobre transparencia y acceso a la información, para la profundización democrática de las sociedades modernas, en el contexto de las Tecnologías de la Información y la Comunicación. Para ello, se aplica una metodología de carácter analítico e interpretativo. Uno de los resultados más destacados en este estudio es el comportamiento de los ciudadanos y ciudadanas que integran esas sociedades, en relación con el uso o desuso de los mecanismos que las legislaciones sobre transparencia ponen a su alcance.

En este sentido, la principal conclusión es que, aun cuando las leyes de transparencia mantienen altos estándares de calidad en Latinoamérica, el ciudadano común no ha desarrollado un interés genuino en la participación activa como destinatario de dicha legislación, ni alcanza a comprenderla como elemento democrático fundamental.

Palabras clave: Transparencia; Estado; sociedad; redes sociales; democracia.

1. Doctora en Comunicación y Periodismo por la Universidad Autónoma de Barcelona, Magister en Periodismo Investigativo, Datos y Visualización por la Universidad Internacional de la Rioja (2017). Licenciada en Comunicación Social por la Universidad Politécnica Salesiana (2005). Es directora y docente de la Carrera de Comunicación Social de la Universidad Politécnica Salesiana (UPS), sede Quito. Pertenece al grupo de investigación de la Universidad Politécnica Salesiana CODEPO y es investigadora del Laboratorio de Periodismo y Comunicación para la Ciudadanía Plural de la Universidad Autónoma de Barcelona. Correo electrónico: nmedranda@ups.edu.ec

2. Doctora en Innovación Educativa y Aprendizajes a lo largo de la vida en la Universidad de Deusto (España) Magister en Ciencias de la Educación PUCE-Ecuador. Licenciada en Ciencias de la Educación, PUCE. Técnica en Comunicación radiofónica para el Desarrollo en la Universidad del Azuay. Especialista en formación $\mathrm{Hu}$ mana Integral IFHIM (Canadá). Profesora agregada PUCE de las materias Innovación educativa, Proyecto integrador l, Axiologia y Ética profesional. Coordinadora general de la Corporación para la Educación Audiovisual Francisco Xavier (CEAFAX). Veinte años de trayectoria en coordinación y ejecución de proyectos educomunicacionales, Realizadora de recursos multimediáticos con fines educativos: vídeos, audios, cds interactivos entre otros. Correo electrónico: vdpalacios@puce.edu.ec 


\section{A look at the society of transparency in Latin America}

\section{Abstract}

This research seeks to analyze, from a critical point of view, the effectiveness of legislation on transparency and access to information, for the democratic deepening of modern societies, in the context of Information and Communication Technologies. To do this, a methodology of analytical and interpretative nature is applied. One of the most outstanding results in this study is the behavior of the citizens who make up these societies, in relation to the use or disuse of the mechanisms that transparency laws put at their disposal. In this sense, the main conclusion is that, even though transparency laws maintain high quality standards in Latin America, ordinary citizens have not developed a genuine interest in active participation as a recipient of said legislation, nor can they understand it as a democratic element. fundamental.
Keywords: Transparency; State; society; social networks; democracy

\section{Introducción}

a transparencia es un asunto antiguo. En su literatura oficial, imperios tan remotos como los de China y Grecia ya hacían referencia a la transparencia, que entendían como el acceso por parte de todos los ciudadanos a "leyes escritas". El hecho de que fueran escritas determinaba primero que las leyes no estuvieran a merced del capricho de los funcionarios y ejecutores del gobierno del Estado, por muy elemental que fuera este, y que estuvieran a disposición de los ciudadanos a los que gobernaban. Era una cuestión fundamental que "confería confianza y una mayor seguridad en la vida cotidiana" (Medranda, 2017: 28) a todos esos ciudadanos o súbditos.

Ya en la época moderna, «la primera nación que promulgó una ley prometiendo acceso público a información oficial fue el Reino de Suecia en 1766» (Orme, 2017: 11). Sin embargo, y según el autor citado, fue a finales del S. XX cuando alcanzó a otros países la preocupación por la información pública. A partir de la ley sueca otros países aprueban también sus propias leyes de transparencia. Estados Unidos aprobó la Ley de Libertad de la Información en 1966, mientras que Canadá la aprobó en 1983. Dos años después, en 1985, Colombia se convirtió en el primer país latinoamericano en contar con una Ley de Transparencia, la misma que fue incluida en la Constitución de la República de Colombia. Otros países en Latinoamérica también promulgaron sus leyes, tal como lo establece Medranda (2017) citando a (http://www.rti-rating.org/country-data/).

A inicios del siglo XXI, 16 países de América Latina ya habían adoptado políticas de transparencia y acceso a la información: Panamá y México (2002); Perú, San Vicente y Granadina (2003); República Dominicana, Argentina y Ecuador (2004); Honduras (2006); Nicaragua (2007); Chile, Guatemala y Uruguay (2008); Brasil y El Salvador (2011) y Guyana (2013) (Unesco, 2012). Como se puede evidenciar, la región ha hecho un importante 
avance en el campo de la legislación respecto del acceso a la información para la ciudadanía. (Medranda, 2017: 33)

En este sentido, en Latinoamérica únicamente cuatro países carecen de una ley de transparencia que garantice el acceso a la información pública: Bolivia, Costa Rica, Cuba y Venezuela. Según Orme, "América Latina ha avanzado más en este campo que cualquier otra región de países en vías de desarrollo, y en ciertos aspectos más que los países de la Unión Europea" (Orme, 2017: 11).

Gráfico 1. Leyes de transparencia en Iberoamérica

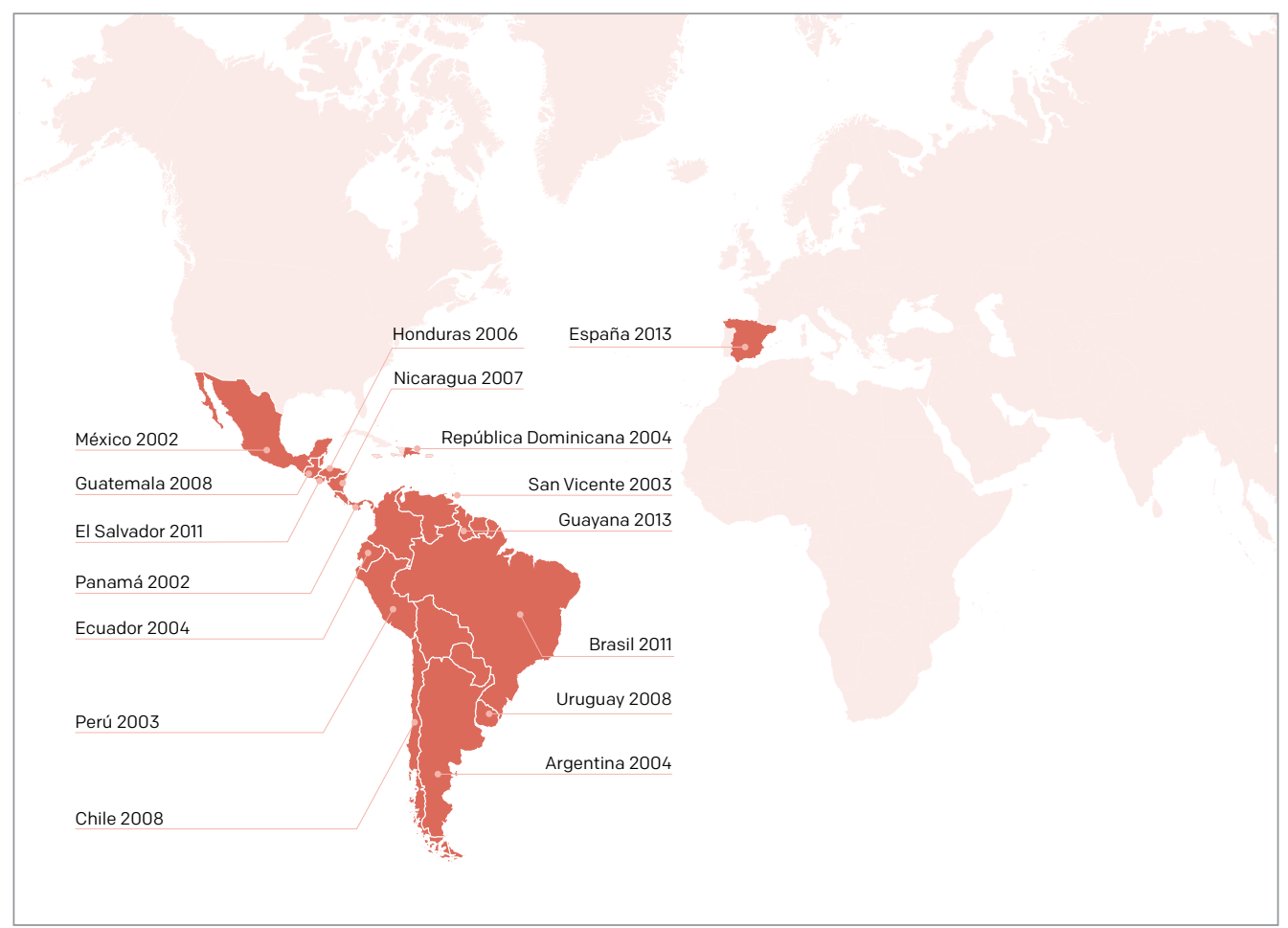

Fuente. Elaboración propia

\section{Metodología}

La metodología aplicada en esta investigación es de carácter analítico e interpretativo, ya que se busca realizar la "descomposición de un fenómeno en sus elementos constitutivos" (Lopera, et al, 2010) e interpretarlos. Para ello, se realiza un estudio completo de lo que está pasando en Latinoamérica en el tema de transparencia, a través del análisis del siguiente marco referencial.

\section{Marco teórico}

El estado de la transparencia en Latinoamérica se puede medir a través de algunosinformes oficiales como el Barómetro Global de la Corrupción, elaborado por Transparencia Internacional, el informe de la UNESCO sobre las condiciones de acceso a la información emitido en 2017, por medio de sus Cuadernos de Discusión sobre Comunicación e Información, y el informe especial sobre corrupción y opacidad en Latinoamérica de 2016 del Centro de Liderazgo a través del Conocimiento, de la firma Llorente \& Cuenca. Los resultados de estos tres informes han sido claves para realizar este análisis, además de varios datos estadísticos de Transparencia Internacional y del Centro para la Ley y la Democracia (IT y CLD, respectivamente, por sus siglas en inglés).

\section{La transparencia en la agenda mundial}

En 2015, la ONU aprobó por unanimidad la agenda 2030, con los diecisiete Objetivos de Desarrollo Sostenible (ODS). En el décimo sexto objetivo planteó "Promover sociedades pacíficas e inclusivas para el desarrollo sostenible, facilitar el acceso a la justicia para todos y crear instituciones eficaces, responsables e inclusivas en todos los niveles" (ONU, 2015: Art.16). Dentro del Art. 16, el apartado 16.10 exige "Garantizar el acceso público a la información y proteger las libertades fundamentales, de conformidad con la legislación nacional y los acuerdos internacionales". Y para evaluar hasta qué punto se establecen dichas garantías en un país, la ONU determinó dos indicadores:

1. "Número de casos verificados de homicidio, secuestro, desaparición forzada, detención arbitraria y tortura a periodistas [y otros] miembros asociados a los medios de comunicación, sindicalistas y defensores de los derechos humanos, en los 12 meses anteriores" (ONU, 2015: art.16.10.1). De acuerdo con la $\mathrm{ONU}$, con este indicador se podrá establecer un barómetro del estado de las libertades fundamentales en cada país.

2. "Número de países que adoptan y aplican garantías constitucionales, estatutarias y/o políticas para el acceso del público a la información" (ONU, 2015: art.16.10.2). Es decir, un indicador del acceso a la información a escala global. (ONU, 2015: Art.16).

Según informa Orme "a finales de 2016, 109 de los 193 Estados miembros de la ONU habían adoptado leyes para garantizar el acceso público a la información, la mayoría cumplió con el objetivo de publicar las leyes de transparencia en los últimos diez años" (2017: 12). En el espíritu de la Agenda 2030 de la ONU, en el Art. 16.10 de los ODS, se recoge la esperanza de que el compromiso con el derecho al acceso a la información, 
que como vemos todavía no es universal, lo sea antes del año 2030. En todo caso, 109 de los 193 Estados de la ONU ya se han comprometido a lograr esos 17 objetivos de desarrollo para 2030. Los avances en este sentido serán monitoreados por la UNESCO, la cual, además, tendrá la responsabilidad de informar periódicamente sobre los logros de la Agenda.

\section{Mediciones internacionales}

La finalidad del Centro para la Ley y la Democracia (CLD), una organización internacional afincada en Canadá que brinda servicios legales con expertos a nivel mundial, es promover, proteger y desarrollar aquellos derechos humanos que sirven de base a la democracia, incluidos los derechos a la libertad de expresión, al voto, a la participación en la gobernanza, al acceso a la información y a la libertad de reunión y asociación.

A partir del 28 de septiembre de 2011 (Día Internacional del Derecho a la Información), la CLD realizó un estudio global con el fin de determinar qué países han establecido una legislación más eficaz para facilitar a la ciudadanía el derecho a la información. Su Global Right to Information Rating contiene 61 indicadores, entre los que destacamos a modo de ejemplo los siguientes:

- Indicador 1: Marco legal con el cual se reconocen los derechos fundamentales de la ciudadanía, entre ellos el de acceso a la información.

- Indicador 4: Alcance del derecho a presentar solicitudes de información. ¿Son todos los ciudadanos, incluso extranjeros y personas jurídicas, quienes detentan ese derecho?

- Indicador 5: A qué información concreta se aplica el derecho de acceso. Es decir, si se aplica a todo el material conservado por o en nombre de las autoridades, en cualquier formato, independientemente de quién lo haya producido.

- Indicador 7: A quién se excluye, si es que se excluye al alguien o a algún organismo del Estado, de la obligación de ofrecer toda la información a la ciudadanía. Es decir, si ese derecho de acceso se aplica tanto al poder Ejecutivo, sin excluir a ninguna clase de organismo, incluidos ejecutivos del gabinete y la administración, todos los ministerios y departamentos, los gobiernos locales, las escuelas públicas, los organismos de salud pública, la policía, las fuerzas armadas, los servicios de seguridad y los organismos de propiedad o los controlados por los anteriores (Mendel, 2010)
La CLD valida los indicadores anteriores y añade otros 57; además, ha creado un parámetro de un valor máximo de 150 puntos, que pueda alcanzar un país que cumpla con todos los criterios en materia de transparencia y acceso a la información. ${ }^{3}$

Como se puede observar, el CLD califica a México como el país del mundo con la mejor legislación en materia de transparencia, tan sólo por debajo de Afganistán mientras que Austria aparece como el país peor calificado. En Latinoamérica, después de México, son Brasil, Colombia y Nicaragua los países con las mejores leyes y mecanismos de transparencia. Todos ellos comparten afinidades histórico-políticas, en especial la de haber sufrido regímenes autoritarios durante el siglo XX, que fueron confrontados por una sociedad civil, respaldada en mayor o menor medida por los medios de comunicación e instituciones independientes que luchaban, entre otras cosas, por una mayor transparencia en las prácticas gubernamentales.

Si bien existe una lógica según la cual la transparencia favorece "tendencias democráticas que apuntan a que el poder sea más público y, en consecuencia, más responsable ante el motivo central de su existencia: los ciudadanos" (Uvalle, 2016: 200), sin embargo, los resultados que arroja el barómetro del Centro para la Ley y la Democracia no solo no parecen sostener esta lógica, sino que incluso pareciera que la contradicen, así por ejemplo: Austria encabeza la lista de los países con peor legislación en materia de transparencia, lo que lo situaría entre los más opacos, y aun así, Viena sigue siendo por noveno año consecutivo la ciudad con la mejor calidad de vida del mundo ${ }^{4}$. Además, según Transparencia Internacional, (2017) Austria se sitúa en el puesto número 16, con una puntuación de 75 sobre 100 del IPC (Índice de Percepción de la Corrupción) en términos de percepción de ausencia de corrupción, según esta medición Ecuador en este mismo año se ubicó en el puesto 120 de 176 países, con un puntaje de 34 en la escala de 100. "De lo que se interpreta que es un país con un alto índice de corrupción en la percepción de los ciudadanos” (Medranda, 2017: 37).

Por otro lado, Transparencia Internacional (2017) posiciona a México en el número 135, con una puntuación IPC de 29 sobre 100. Los resultados del Índice de Percepción de la Corrupción revelan cómo la aplicación de estas leyes no están en consonancia con la calidad de las mismas, así por ejemplo, la legislación de México, ejemplar a escala global en materia de transparencia y acceso a la información, debería repercutir directamente en procesos democráticos tales como fomentar el debate público y la participación ciudadana y, en última instancia, repercutir considerablemente en la mejora de la vida de sus ciudadanos y ciudadanas. Estas contradicciones estadísticas nos obligan a considerar como prioritario el diseño de procedimientos que tengan en

3. Fuente: Center of Law and Democracy (https://www.law-democracy.org/live/)

4. https://www.mercer.es/sala-de-prensa/estudio-calidad-vida-2018.html 
cuenta en primer lugar, la Ley y segundo, el cumplimiento de los derechos (Molina, Simelio y Corcoy, 2017).

En este sentido, los organismos de transparencia, igual que las leyes que promueven, surgen a partir de una necesidad convertida en derecho: la necesidad del ciudadano de acceder a la información de las instituciones y conocer como mínimo, en sus aspectos fundamentales, aquellas cuestiones que le afectan directamente en su vida cotidiana: transparencia económica, organizativa y de ejecución. Se espera que esto permita al ciudadano, precisamente, ser un participante activo en la vida política del país, de forma que pueda tomar decisiones (como por ejemplo por quién o por qué partido votar en las elecciones) con conocimiento de causa.

La transparencia debe funcionar como un mecanismo que cumple con una triple función: dar acceso a la información pública de forma sistemática, empoderar al ciudadano, y construir una democracia participativa (López y Medranda, 2016). Esta triple función, debe servir para mejorar la democracia y la vida de los ciudadanos y las ciudadanas, objetivo último, "motivo central" de la vida pública y del Estado. Sin embargo, al analizar los criterios de la CLD, se observa que los países con leyes más eficientes (en sus recursos, procesos y ejecución) no son precisamente los más eficaces para cumplir con esa lógica según la cual, a mayor transparencia, mayor calidad democrática y mejor calidad de vida.

Así pues, podemos concluir que no basta con tener acceso a una gran cantidad de datos para alcanzar esa triple función de la transparencia. Será necesario también tener acceso a los antecedentes y al contexto desde el que se generaron esos datos, pues la legislación a favor de la transparencia, efectivamente "permite el acceso a las decisiones o a los datos y/o estadísticas que fundamentan las mismas, pero no es posible conocer el cómo y el porqué de dichas decisiones: quiénes se reunieron, con qué fin y en qué circunstancias" (Herrero y López: 35).

Esta inconsistencia en los mecanismos del derecho a la información se reproduce en casi todos los países latinoamericanos que cuentan ya con una ley de transparencia. Así, por ejemplo: En Ecuador, la Ley Orgánica de Transparencia y Acceso a la Información Pública (LOTAIP), fue aprobada en mayo de 2004. Esta Ley presenta algunas flaquezas (...), primero porque esta ley responde a la Constitución de 1998 y no tanto a la actual Carta Magna, aprobada en 2008, [y] segundo, porque no incluye (...) la publicación de información sobre las declaraciones juramentadas de bienes de los políticos, hecho que se deja exclusivamente en manos de la Contraloría del Estado" (Medranda, 2017, pág. 35).
Cabe destacar que, no basta con que las leyes de transparencia sean eficientes en el papel, sino que debe haber además una mayor eficacia en su procesamiento y en su ejecución. 0 , dicho de otra forma, no deben quedarse en una mera declaración de intenciones para cubrir el expediente que marcan aquellos ODS de la ONU, sino que deben implementarse materialmente, de forma exhaustiva y sin lagunas, hasta sus últimas consecuencias. Las leyes de transparencia carecen de efectividad por sí mismas.

Es necesario llevarlas a la práctica desde organismos del Estado independientes de los poderes a los que deben fiscalizar. Esos organismos independientes, respaldados por dicha legislación, velarían por la adquisición y posterior publicación de la información sensible. Para crear esos organismos, por un lado, se deben dotar partidas presupuestarias suficientes, y por otro se debe nombrar y poner a su frente a personal independiente, similar en su función y su funcionamiento a los fiscales o los inspectores de otras instituciones del Estado. Pero, además, sería de esperar que el libre acceso a la información permitiera a la ciudadanía involucrarse en el proceso y, a su vez, que los organismos independientes pudieran favorecer el debate público, el plebiscito, que tuvieran acceso a los medios de comunicación de masas, con el fin último de que la toma de decisiones colectivas fuera consciente, bien informada y apegada a los fines democráticos.

\section{Transparencia y rendición de cuentas}

El sentido de comunidad es un factor fundamental para que el acceso a la información cobre una dimensión colectiva, alejada del individualismo y el aislamiento. Sin ese sentido de comunidad, la transparencia no tendría la dimensión pragmática que debe tener, como proceso de capacitación para la participación ciudadana. Se trata del sentido que permite a los ciudadanos vertebrarse a través de organizaciones civiles, redes de apoyo y comités ciudadanos.

Uno de los problemas que subyacen, es la falta de cohesión social en Latinoamérica, se podría decir que es el primer impedimento para que se consolide esa dimensión pragmática de la transparencia, cuya principal vertiente es la rendición de cuentas de los gestores públicos ante los ciudadanos, en relación con su gestión particular de lo público. Al fin y al cabo, la información y las cuentas se refieren, en la práctica, a la cosa pública, a lo común, a la generalidad, a lo que se comparte y se financia con la aportación de cada uno de esos ciudadanos y ciudadanas.

En relación con esta cuestión, no es posible soslayar que los procesos democráticos son en Europa muy diferentes de los de nuestro continente, empezando por la brecha de desigualdad que impera en muchas de sus regiones, en aumento año tras año según algu- 
nos indicadores. No es casualidad que, en las últimas décadas, a partir de los años 80 para ser específicos con la apertura, la liberalización comercial, los efectos de la globalización y la cohesión social hayan pasado a un primer plano en el debate público, y hayan adquirido gran relevancia en nuestra sociedad.

En 2005, el Consejo Europeo definió la cohesión social como la "capacidad de la sociedad para asegurar el bienestar de todos sus miembros, incluyendo el acceso equitativo a los recursos disponibles, el respeto por la dignidad humana, la diversidad, la autonomía personal y colectiva, la participación responsable y la reducción al mínimo de las disparidades sociales y económicas" (Torno, et al., 2015: 25) Así, una sociedad cohesionada es una comunidad cuyos miembros se benefician de su pertenencia, en la medida en que todos procuran asegurar el bienestar del otro, sin distinciones, de forma que el "nosotros" alcanza preeminencia sobre el "yo".

Todos sus integrantes persiguen de esa manera metas comunes por medios democráticos, y a través del apoyo mutuo. En esa comunidad cohesionada las desigualdades sociales y económicas se habrían reducido drásticamente.

Sin embargo, "los distintos mecanismos de transparencia pueden incrementar la legitimidad democrática [de los poderes públicos] y también ayudar a que los ciudadanos se transformen en participantes activos, no reactivos, en los asuntos públicos" (Martínez, 2005). Es decir, la "participación responsable" y la "legitimidad democrática" también serían factores de cohesión social. Y puesto que esta participación y esta legitimidad se consiguen, entre otras cosas, mediante políticas de transparencia y de rendición de cuentas, hemos de concluir que dichas políticas redundarían en esa deseable cohesión social. Pero parece que en Latinoamérica, donde se dispone de leyes modelo en materia de transparencia, o bien estas no ayudan a la cohesión social, o bien las enormes distancias económicas y socioculturales de sus regiones, es decir, la falta de esa cohesión, dificulta la implementación de políticas de transparencia, de las cuales una de las más relevantes para los ciudadanos es la rendición de cuentas. Así pues, estaríamos ante un círculo vicioso en el que la falta de transparencia produciría falta de cohesión, y la falta de cohesión, indiferencia por la transparencia.

\section{La corrupción como mal endémico del continente}

La definición de "corrupción" más aceptada por académicos y organismos sociales es la utilizada por Transparencia Internacional. Esta institución la define como "el mal uso del poder encomendado, para obtener beneficios privados" (Pope, 2000). Incluye hechos como el soborno, el peculado, el tráfico de influencias, la omisión y el nepotismo, y en todos los casos tiene como consecuencia mermas y desvíos del peculio del Estado y, por ende, del de los ciudadanos que reciben servicios y prestaciones de este. Pese a los esfuerzos realizados en transparencia para evidenciar estos ilícitos en las prácticas políticas y facilitar su denuncia la corrupción sigue siendo un fenómeno global, aunque con distinto alcance según las regiones. Así, no es posible soslayar que en Latinoamérica el fenómeno llega a grados extremos y por lo tanto alarmantes.

Desde 2015, la corrupción ha cobrado una gran notoriedad en América Latina por la magnitud de los casos que han salido a la luz; una corrupción que sigue muy presente aún en esa región pese a que muchos aspectos han cambiado sustancialmente en los últimos años, en los que se ha creado un entramado institucional que, con sus defectos y sus virtudes, sus fortalezas y debilidades, sirve para controlar y perseguir la corrupción (Desarrollando ideas, 2016, pág. 02).

En este sentido, el Banco Mundial calculó en el año 2013 que entre 2001-2002 se pagaron sobornos, tanto en los países en desarrollo como en los desarrollados, por una cantidad de un trillón de dólares, el 3\% del PIB mundial. Según el Banco de México, la corrupción en el país representa cerca del 9\% del PIB. La Federación de Industriales del Estado de Sao Paulo estimó que la corrupción costó en 2010 entre el 1,4 y el 2,3\% del PIB brasileño. En Perú, según la ex Primera Ministra Ana Jara, llegaría al 2\% del PIB. En Guatemala, según estimaciones de la ONG Acción Ciudadana, se perdieron en prácticas corruptas alrededor de 12 mil millones de quetzales del Presupuesto General de la Nación de 2015, de los que, entre el 25\% y el 30\% estaba destinado a inversión pública (Desarrollando ideas, 2016, pág. 03).

Como puede observarse, México y Nicaragua se encuentran a la cabeza de la lista de los países latinoamericanos más corruptos, países que la CLD destaca al mismo tiempo por disponer de las mejores leyes de transparencia y acceso a la información del mundo. Venezuela es la peor calificada a este respecto, situada en el puesto 166 de la tabla. Este país subió casi 10 posiciones en el ranking internacional, pues en 2015 se encontraba en el puesto 158. Pero Venezuela no manifiesta un aumento tan preocupante como el de Nicaragua, que ascendió 22 puestos, al colocarse en el lugar 145

Sólo dos países, Uruguay, en la posición 21, con 71/100 puntos de valoración positiva, y Chile, en la 24 con una ratio de 66/100, alcanzan índices tan positivos como los que se encuentran en los países de la OCDE. Pero el índice de la CLD, como ya hemos señalado, presenta una marcada heterogeneidad de parámetros. Hay algunos casos que llaman poderosamente la atención. México, por ejemplo, se ubicaba en 2015 en el lugar 95, y en 2016 llegó a la posición 123 de un total de 176 países evaluados. Obtuvo 30 sobre 100 puntos en la escala internacional, donde 100 representa la mejor evaluación en la mate- 
ria. "40 posiciones separan a México de China, India y Brasil, sus principales competidores económicos. Entre las 35 economías que integran la Organización para la Cooperación y el Desarrollo Económicos (OCDE), México se ubica en el último lugar." ${ }^{5}$

A diferencia de Europa Central, donde la percepción de la corrupción es mínima, en Latinoamérica el fenómeno es mucho más complejo. La naturaleza sistémica de la corrupción en las sociedades de este ámbito geográfico la convierte en un hecho culturalmente inherente a los procesos políticos y, por tanto, encuentra una mayor aceptación en ellas. Así, la percepción social la considera como un mal permanente e incluso necesario para la supervivencia, sustentado desde las esferas del poder y generalizado de tal manera que combatirlo resulta casi imposible.

La corrupción se beneficia así de una especie de consenso tácito, y su ejercicio se acepta, no como un acto ilícito, sino como parte de los usos y costumbres del lugar. Esta "corrupción gris" en una sociedad en la que no están bien establecidas las distancias entre lo bueno y lo malo, se convierte en un elemento del paisaje, por lo que tomar medidas para atajar el fenómeno se convierte en una tarea extremadamente difícil. En «El que a buen árbol se arrima...» intercambio de favores y corrupción (Zalpa, Tapia y Reyez, 2013), los autores señalan la corrupción en México (y por su relación histórica en gran parte de América Latina) como una manifestación cultural de mucho arraigo cuyo origen se pierde en el tiempo. Estos autores llaman la atención en su obra sobre el hecho de que "se ha instalado un 'sentido común' que hace de la corrupción parte de las conductas aceptables", y que "en pocas palabras, los mexicanos creen que no es que uno quiera ser corrupto, lo que pasa es que resulta inevitable si se vive en este país (50\%)". Y este mismo fenómeno sucede en toda Latinoamérica.

Tomemos como ejemplo los índices de soborno. Estos varían sustancialmente de un país a otro. Cerca de la mitad de los ciudadanos de México y de la República Dominicana afirman haberlo pagado al menos una vez en los 12 meses anteriores (el 51\% y el 46\%, respectivamente). En cambio, Trinidad y Tobago muestra el índice de soborno más bajo de los países encuestados. Allí, solamente el 6\% de las personas en contacto con un funcionario público en los 12 meses anteriores pagó un soborno (Pring, 2017, pág. 14). Mientras que, en Ecuador, el 28\% de los ecuatorianos que habían realizado un trámite público en el año 2017, pagó soborno.

Normalizar lo ilícito y encubrirlo con falacias que apelan a lo emocional y a un "sentido común" un tanto especial, son dos de los subterfugios que utilizan corruptores y corrompidos para generar un sentido de identificación del resto de ciudadanos y de ciudadanas con

5. https://www.animalpolitico.com/2017/01/corrupcion-indice-transparencia-mexico/ ellos, en una sociedad donde la omisión, el favoritismo, el intercambio de favores y el nepotismo se confunden con la solidaridad, y el soborno con el apoyo o la propina. Además, los actos de corrupción en las clases bajas se enmascaran bajo la falacia ad hominem de que la persona se ha corrompido por necesidad. De esta manera, la "corrupción gris" es una cadena que se extiende a toda la sociedad y que abarca el mayor espectro posible por no tener unos límites claros.

En este escenario es difícil plantear una relación virtuosa entre el acceso a la información y la rendición de cuentas, y entre la democracia y la calidad de vida, ya que no parece existir una verdadera interacción entre ambos extremos, toda vez que, para el ciudadano latinoamericano, tener acceso a información del Estado y sus instituciones (una información como no la tienen, por ejemplo, en Austria) no le ha supuesto un beneficio directo en términos de democracia, justicia y bienestar.

La expresión "información es poder" no se aplica a una relación donde dicha información no es procesada y analizada para un fin común que motive a la ciudadanía a reaccionar en consecuencia. Lo que sí puede observarse de manera puntual a través de las redes sociales es que la disponibilidad de información no parece orientar a los ciudadanos a ejercer prácticas democráticas como son el debate y la toma de decisiones, o despertar en ellos una justa indignación ante determinados problemas y la posterior exigencia de respuesta a los poderes para que los solucionen. En cambio, dicha información acaba por enfangarse, en las redes sociales, en el terreno del espectáculo y, en ocasiones, en el de lo absurdo.

\section{La infoxicación en la era digital}

En la actualidad, gracias a las Tecnologías de la Información y la Comunicación, los ciudadanos y las ciudadanas reciben constantemente y de forma inmediata, avalanchas de información. Esa constancia y esa inmediatez de gran cantidad de datos, sin embargo, impiden que tengan tiempo para la reflexión y el diálogo sobre la información que reciben. Detrás de una oleada sobreviene otra oleada igual o más intensa que la anterior, generando un exceso de datos que termina por intoxicar al ciudadano. Escribe Paz (1995) en su prólogo al libro Las Enseñanzas de Don Juan (Castaneda, 1968), que "la mucha luz es como la mucha sombra: no deja ver", y también que "las obras son literalmente deglutidas, ya que no gustadas, por lectores apresurados y distraídos".

La "sobrecarga informativa" (Toffler, 1971) se entiende como el exceso de información a la que se ve expuesto un usuario, principalmente un "cibernauta". Este usuario o "cibernauta", al no poder procesar esa "sobrecarga", acaba por sucumbir ante una sensación 
paralizante de frustración y angustia. Estará informado de todo pero enterado de nada. La sobreexposición a los datos sin ningún filtro de calidad, carente por completo de organización según su importancia, congestiona la capacidad de procesamiento del sistema nervioso de sus destinatarios. Se tienen nociones de todo o de casi todo, sin profundizar en ningún asunto porque no hay tiempo material para ello. La ingesta de datos es continua y compulsiva, inhibiendo la capacidad de discernir y generando un deseo inalcanzable por absorber toda la información y no perderse de nada. La consecuencia directa de ello es en un primer momento, como decimos, la frustración y la parálisis, la confusión y la incapacidad de reflexión y de decisión. Posteriormente, en una especie de ejercicio de supervivencia, la indiferencia. Es esta la que podríamos llamar una "enfermedad moderna", que afectará crecientemente a los jóvenes que despiertan a la vida con un terminal celular entre las manos, y menos a los adultos y a los ancianos, que han sido testigos del paso de sociedades analógicas a sociedades digitales

\section{El individuo en la sociedad de la transparencia}

"Cuando yo era joven, había una diferencia importante entre ser famoso y estar en boca de todos. Muchos querían ser famosos por ser el mejor deportista o la mejor bailarina, pero a nadie le gustaba estar en boca de todos por ser el cornudo del pueblo o una puta de poca monta... en el futuro esta diferencia ya no existirá: con tal de que alguien nos mire y hable de nosotros, estaremos dispuestos a todo." (Umberto Eco, 2016)

De acuerdo con Umberto Eco (2016), el individuo en la sociedad de la transparencia es un narcisista social. Este se encuentra sumergido en la admiración de su "YO", preocupado por mostrarse a los demás, como hombres y mujeres que viven en el paraíso, donde no existe más que la perfección. El filósofo Byung-Chul Han, expone que esta exposición del hombre perfecto elimina cualquier contradicción en la comunicación, la acelera en este sentido, ya que ante la ausencia de un "No" que se oponga en ella a alguno de sus vectores, acaba por convertirse en un proceso vacío, no dialéctico, convertida indefinidamente en un flujo positivo. Así, considera que la exposición en una comunicación de este tipo es pornográfica, porque el individuo busca desnudarse en su totalidad, exponer su imagen sin reserva ante los otros "yoes" que le evalúan y que al mismo tiempo compiten por superarse en el estatus positivo-expositivo, en una persecución obscena, casi onanista, de autoafirmación. (Han, 2014).

Esta exposición positiva del ser humano aleja al ciudadano del "Yo social", ya que se ocupa de sus objetivos individuales. En este sentido, el individuo se separa de la comunidad a la que pertenece sin darse cuenta, porque sus decisiones y acciones están relacionadas en un
"YO" para sí mismo, los demás no están involucrados en su quehacer. Es un "Yo" para sí mismo que se mira en el espejo de otro "Yo", en el que la repercusión de los acontecimientos de orden social (salud, política, educación, seguridad, transparencia, etc.) pasa a un plano irrelevante, es decir, se convierten en un mero escenario, como el decorado en el cual se expone ese "Yo". Ya no se trata entonces de lo que sucede en la comunidad, en el vecindario, en su ciudad, en su país, y cómo le afecta a él y a los demás, sino cómo es expuesto, cómo le miran, cuántos seguidores le miran, en una comunidad virtual a la que en el fondo no pertenece.

La comunidad y todo lo que implica (la sociedad y el Estado) pertenece así a un "ello" ajeno a las circunstancias inmediatas y aceleradas de su experiencia personal. Pierde relevancia lo que sucede en la comunidad, en tanto no tenga una relación directa con su proyección y su exposición.

Es por situaciones como las expuestas en los párrafos anteriores, que el tema de la transparencia pasa desapercibido en la sociedad actual, el individuo que se preocupa del "YO" individual, no vela por el "NOSOTROS", por lo tanto, no exige a sus mandantes información completa y veraz, para auditar al gobierno, porque su tiempo está centrado en obedecer a sus necesidades individuales.

Mientras tanto, los políticos de turno aprovechan la distracción social, y le dan más show para entretenerlo, de este modo, el individuo de la sociedad actual, no se preocupa por exigir información contrastada y contrastable, ni los representantes políticos ponen los medios para que el ciudadano común pueda comprender, lo que implica que: "La información debe estar presentada en formatos periodísticos que permitan la lectura rápida y por ende la comprensión de la información en su totalidad. Sólo de esta manera las personas tendrán una oportunidad real de participar con fundamentos" (Medranda, 2017: 78). El hecho de contar con información completa al alcance de toda la ciudadanía permitirá que el "YO" social, se preocupe por un "NOSOTROS".

\section{Conclusiones}

En tanto el ciudadano carezca de un interés proactivo en los asuntos públicos, los esfuerzos de los gobiernos, las ONG's y otros organismos interdependientes por ejercer la transparencia, no podrán alcanzar sus objetivos ni los resultados que persiguen.

En la medida en que el ciudadano ha canjeado el interés proactivo en los asuntos públicos por unos intereses individuales inmediatos, la población en la que se integra carece de empuje social. Esa población ya no funciona como una masa, sino como un enjambre que "zumba" momentáneamente ante un estímulo, hasta que este pierde 
fuerza y es sustituido por el siguiente, y así sucesivamente ad infinitum. Incluso cuando los conflictos sociales requieren de un amplio rango de análisis y por ende, de tiempo de reflexión, para el ciudadano pierden interés en tanto que cada minuto aparece un conflicto más novedoso que se solapa y tapa los anteriores.

El exceso de información mediática convierte las noticias en espectáculo, distrayendo al ciudadano de los temas esenciales, aquellos que le conciernen directamente. La opinión pública se convierte en una boca sin fondo que lo traga todo sin procesar nada.

La relación entre la transparencia, el acceso a la información y la acción ciudadana requiere de una mirada que profundice en el fenómeno psicosocial de lo que el individuo experimenta hoy día.

Es necesario reflexionar sobre la ingente cantidad de datos y de información producida por las instituciones, por los medios y por la sociedad en general, y orientar los esfuerzos de las instituciones de transparencia hacia las necesidades del ciudadano.

Sin duda será un reto ampliar los estudios sobre el fenómeno de las redes sociales y su influencia en la toma de decisiones de los ciudadanos y las ciudadanas, pero ello permitirá aprovechar los mecanismos tecnológicos de una manera más eficiente para fortalecer la rendición de cuentas y, de forma sistemática, encaminar los hábitos de consumo de información de aquellos y aquellas hacia la proactividad cívica.

\section{Referencias}

De los medios y la Comunicación de las organizaciones a las redes de valor. Actas del II Simposio de la Red Internacional de Investigación de Gestión de la Comunicación, XESCOM (pág. 1004 a 1018). Obtenido de https://xescom2016.wordpress.com/libro-de-actas/

Desarrollando Ideas. (2016). La corrupción, el talón de Aquiles de las democracias latinoamericanas. Informe Especial. Recuperado de: https://www.desarrollando-ideas.com/ wp-content/uploads/sites/5/2016/09/160912_DI_informe_Corrupcion_LatAm_ESP.pdf

Eco, U. (2016). De la Estupidez a la Locura. Obtenido de ePubLibre: https://www.epublibre. org/libro/detalle/34846

Escamilla, M. (2011). Aplicaciones básicas del método científico: modelo interpretativo. Recuperado de: https://www.uaeh.edu.mx/docencia/VI_Presentaciones/licenciatura_en mercadotecnia/fundamentos_de_metodologia_investigacion/PRES42.pdf

Han, B. (2013). La Sociedad de la Transparencia. Recuperado de: https://www.epublibre.org/ libro/detalle/24443
Han, B. (2014). Psicopolítica. Obtenido de: https://www.epublibre.org/libro/detalle/23466.

Herrero, A., y Lopez, G. (2016). Acceso a la información y Transparencia en el Poder Judicial. Recuperado de: http://siteresources.worldbank.org/PSGLP/Resources/accesoalainformacionytransparencia.pdf

http://www.onu.org.mx/wp-content/uploads/2017/07/180131_ODS-metas-digital.pdf Innerarity, D. (21 de Junio de 2009). Las transformaciones de lo privado y lo público. Libre Pensamiento (60), pp. 80. Recuperado en: http://librepensamiento.org/archivos/3552

Lopera, Juan,et al. (2010). El método analítico como método natural. Revista Crítica de Ciencias Sociales y Jurídicas, 1-2.

López, P., y Medranda, N. (2016). Transparencia, comunicación institucional e información pública: análisis de las prefecturas de Pichincha, Guayas e Imbabura.

Martínez, A. (2005). La gobernanza hoy: 10 textos de referencia. Recuperado de: https://libros-revistas-derecho.vlex.es/source/gobernanza-hoy-10-textos-referencia-6617 .

Medranda, N. (2017). Calidad y Transparencia en la Información y Comunicación que se emite a través de las páginas webs de los Municipios: Comparación de caso Ecuador y España. Tesis Doctoral, (pp.18 -78).

Mendel, T. (2010) La Metodología de Clasificación Legislativa para el Derecho a la Información en Center for Law and Democracy. Recuperado de http://www.law-democracy.org/live/ wp-content/uploads/2012/08/RTI-en-espanol.pdf

Molina, P., Simelio N., y Corcoy, M. (2017): “Metodologías de evaluación de la transparencia: procedimientos y problemas". Revista Latina de Comunicación Social, 72, pp. 818 a 831. http://www.revistalatinacs.org/072paper/1194/44es.html DOI: 10.4185/RLCS-20171194

ONU. (2015). Metas de los Objetivos de Desarrollo Sostenible (ODS), Objetivo 16. Obtenido de:

Orme, B. (2017). Acceso a la información: Lecciones de la América Latina. Cuadernos de Discusión de Comunicación e Información, (pp. 5-7).

Paz, O. (1995)." La mirada anterior" Prefacio. Las enseñanzas de Don Juan. México: Fondo de Cultura Económica.

Pope, J. (2000). Libro de Consulta 2000 de Transparencia Internacional. International Transparency. Recuperado en: https://www.transparency.org/whatwedo/publication/las_personas_y_la_corrupcion_america_latina_y_el_caribe

Pring, C. (2017). Las personas y la corrupción: América Latina y El Caribe. Berlin: Transparency International.

Toffler, A. (1971). El Shock del Futuro. Recuperado de: https://www.epublibre.org/libro/detalle/36651

Torno, Joaquin, et al. (2015). Transparencia, rendición de cuentas y participación: una agenda común para la cohesión social y la gobernanza en América Latina. Recuperado de: https:// www1.diba.cat/uliep/pdf/52249.pdf

Transparencia Internacional. (2017) Índice de Percepción de la Corrupción. Recuperado de https://transparencia.org.es/wp-content/uploads/2018/02/tabla_sinteti- 


\section{$210 \mid \begin{aligned} & \text { CIUDADANÍAS } \\ & \text { DIGITALES }\end{aligned}$}

ca_ipc-2017.pdf

Uvalle, R. (2016). Fundamentos de la transparencia en la sociedad contemporánea. Revista Mexicana de Ciencias Políticas y Sociales (226), 199-220.

Zalpa, G., Tapia, E., y Reyes, G. (2013). “El que a buen árbol se arrima...” intercambio de favores y corrupción. Cultura y Representaciones Sociales. 09(17) Recuperado de: http://www. scielo.org.mx/scielo.php?script=sci_arttext\&pid=S2007-81102014000200005 